\title{
Recirculación de la sangre durante la sesión de hemodiálisis en el catéter tunelizado Palindrome ${ }^{\mathrm{TM}}$
}

\author{
"Premio Covidien Quinton 2011 al mejor trabajo de investigación sobre catéteres permanentes \\ y la calidad de las sesiones de hemodiálisis"
}

\section{Isabel Crehuet Rodríguez - Pilar Méndez Briso-Montiano - Teresa Mulero San José - María Bernárdez Lemus - Ángel Jiménez Maldonado - Beatriz Toribio Manrique}

\section{Enfermeras/os}

Servicio de Diálisis. Hospital Universitario Río Hortega. Valladolid

\section{Resumen}

Uno de los problemas más frecuentes que encontramos al utilizar los catéteres venosos tunelizados como acceso vascular para hemodiálisis, es un déficit de flujo sanguíneo, teniendo que invertir las líneas del circuito para poder continuar la sesión. Los investigadores, conscientes de que así puede aumentar la recirculación y derivar en una menor calidad de la técnica, han diseñado un nuevo modelo de catéter, para evitar en lo posible la recirculación de la sangre.

Actualmente, este tipo de catéter es el que se implanta a nuestros pacientes; debido a esto y a la escasez de estudios publicados en estos nuevos catéteres sobre este tema, realizamos un estudio para calcular el porcentaje de recirculación que tienen dichos catéteres, tanto con las líneas del circuito en posición normal como en posición invertida. Calculamos este porcentaje analizando la determi-

\begin{tabular}{|c|}
\hline Correspondencia: \\
Isabel Crehuet Rodríguez \\
Servicio de Diálisis \\
Hospital Universitario Río Hortega \\
C/ Dulzaina, 2 \\
47012 Valladolid \\
E-mail: crebel@hotmail.com \\
\hline
\end{tabular}

nación sérica de la urea, en 4 muestras de sangre, extraídas semanalmente, con las líneas del circuito de hemodiálisis en ambas posiciones y aplicamos la fórmula de recirculación:

\section{$R=(B U N$ A2-BUN A1/BUN A2-BUN V) x 100.}

Tras los resultados obtenidos podemos concluir, que el porcentaje de recirculación de la sangre en dichos catéteres es prácticamente el mismo, tanto con las líneas del circuito en una posición como en otra, habiéndose obtenido unas cifras más que aceptables para poder conseguir hemodiálisis de buena calidad, según los parámetros que aconsejan las guías de la sociedad española de nefrología de accesos vasculares.

\section{PALABRAS CLAVE:}

- HEMODIÁLISIS

- ACCESOS VASCULARES

- CATÉTERES VENOSOS CENTRALES

TUNELIZADOS

- RECIRCULACIÓN

- COLOCACIÓN DE LÍNEAS 
Blood recirculation during the haemodialysis session in the tunnelled catheter Palindrome ${ }^{\mathrm{TM}}$

\begin{abstract}
One of the most frequent problems we find when using tunnelled venous catheters as vascular access for haemodialysis is a shortfall in blood flow, making it necessary to invert the circuit lines in order to continue the session. Aware that this can increase recirculation and lead to a decreased quality of the technique, researchers have designed a new catheter model to avoid blood recirculation as much as possible.
\end{abstract}

This is the type of catheter currently used with our patients; because of this and the scarcity of studies published on these new catheters with regard to this matter, we carried out a study to calculate the percentage recirculation of these catheters, both with circuit lines in normal position and in inverted position. We calculated this percentage by analysing the determination of urea in serum, in 4 blood samples drawn weekly, with the haemodialysis circuit lines in both positions, and we applied the recirculation formula:

\section{$R=(B U N$ A2-BUNA1/BUN A2-BUN V) $\times 100$}

Based on the results obtained we can conclude that the percentage of blood recirculation in these catheters is practically the same with the circuit lines in each position, and the figures obtained were more than acceptable to achieve a good quality of haemodialysis, according to the parameters recommended by the spanish nefrology society vascular access guidelines.

\section{KEY WORDS:}

- HAEMODIALYSIS

- VASCULAR ACCESS

- CENTRAL TUNNELLED VENOUS CATHETERS

- RECIRCULATION

- LINE PLACING

\section{Introducción}

El progresivo envejecimiento de los pacientes con enfermedad renal crónica (ERC) junto a su elevada comorbilidad, han hecho que aumenten los problemas para la creación y mantenimiento de las fístulas arteriovenosas (FAVI) utilizadas como acceso vascular para la hemodiálisis (HD). Esto ha dado lugar a que, cada vez, sea mayor el número de sujetos portadores de catéteres venosos centrales, para realizar esta técnica. El empleo de estos catéteres se asocia a un alto riesgo de infecciones y trombosis venosas que condicionan la supervivencia del paciente ${ }^{1}$. Por ello, es de vital importancia el uso de protocolos, generalmente diseñados y aplicados por el personal de enfermería, para reducir en lo posible dichas complicaciones.

A pesar de estas medidas, asistimos con relativa frecuencia a la presencia de problemas, como la disfunción del catéter por insuficiente flujo de salida de la sangre, a través de la rama arterial. Ante esta situación, la forma habitual de actuar es la inversión del flujo en las ramas del catéter, lo que suele ocasionar un aumento en la recirculación del acceso con la consiguiente disminución de la calidad de la sesión de HD $^{1}$.

Por otra parte, sabemos que en los últimos años se han producido importantes avances en el diseño de los catéteres con el objeto de disminuir este problema ${ }^{2,3}$.

Teniendo en cuenta estos hechos, hemos querido analizar en el presente estudio, el grado de recirculación de estos nuevos catéteres tunelizados, implantados en los pacientes de nuestra unidad.

\section{Objetivos}

- Analizar el porcentaje de recirculación de la sangre en los catéteres tunelizados que portan nuestros pacientes, tanto con las líneas del circuito de HD en posición normal como con ellas en posición invertida.

- Valorar si la diferencia de recirculación, entre ambas posiciones, es tan importante como para que nos obligue a modificar otros parámetros de la sesión para conseguir una HD adecuada. 


\section{Material y método}

En nuestra unidad, actualmente, se dializan 43 pacientes, de los cuales $21(48,8 \%)$ son portadores de catéter venoso central para HD.

Los criterios de inclusión en el estudio han sido:

- Portadores de catéteres con el mismo diseño (diámetro de 14,5 French, puntas distales simétricas en forma de espiral en $Z$, tabique central y orificios laterales).

- Catéteres que puedan alcanzar un flujo de sangre de $300 \mathrm{ml} / \mathrm{min}$, con líneas del circuito de HD en posición normal y con ellas en posición invertida.

- Consentimiento del paciente para la realización del estudio.

Antes de comenzar el estudio, nuestros pacientes fueron informados del mismo y se pidió su consentimiento por escrito, siendo aceptado por todos ellos.

Atendiendo a los demás criterios hemos realizado un estudio transversal, observacional, sobre una muestra de 12 pacientes ( $52^{\prime} 1 \%$ de los portadores de catéteres), que cumplían los 3 criterios de inclusión en el estudio.

La no inclusión de los 9 pacientes restantes (47'9\%) fue debida a:

- 4 pacientes no cumplían el primer criterio, de los cuales 2 de ellos tampoco cumplían el segundo.

- 4 pacientes no cumplían el segundo criterio.

- 1 paciente fue excluido por empezar a utilizar otro acceso vascular (FAVI) al poco de comenzar el estudio.

Analizamos un total de 13 sesiones de HD (las correspondientes a un mes) en cada paciente.

En cada sesión se estudiaron:

- Flujo de sangre (Qb)

- Flujo del líquido de diálisis (Qd)

- Presión Venosa (PV)

- Presión Arterial (PA)

- Kt y Kt/v medidos por los monitores

- $\mathrm{N}^{\circ}$ de Infecciones
- $\mathrm{N}^{\circ}$ de disfunciones y aplicación del protocolo de Urokinasa

1. Valoramos el porcentaje de recirculación mediante la determinación analítica de la urea ${ }^{1-4-5}$ realizándolo según indica el siguiente protocolo.

2. Extracción de la primera muestra de sangre en los primeros 30-60 minutos de HD, con el paciente en situación hemodinámica estable y a un flujo de sangre de $300 \mathrm{ml} / \mathrm{min}$.

3. Poner ultrafiltración (UF) $=0$ (UF mínima).

4. Tomar muestras simultáneamente de las líneas del circuito arterial ( $\mathrm{Al}$ ) y venosa (V) en las condiciones anteriormente citadas.

5. Inmediatamente después, bajar el flujo de la bomba a $50 \mathrm{ml} / \mathrm{min}$. y esperamos 60 segundos.

6. Parar la bomba de sangre $(Q b=0)$.

7. Pinzar la línea arterial por encima de la toma de muestras.

8. Tomar una muestra de sangre de la línea arterial del circuito (A2).

9. Invertir las líneas: rama arterial del catéter con línea venosa del circuito y viceversa.

10. Esperar un tiempo de 10 minutos aproximadamente para asegurarnos de que se estabiliza de nuevo la HD.

11. Poner de nuevo flujo de bomba a $300 \mathrm{ml} / \mathrm{min}$ y $U F=0$ ).

12. Repetir los pasos del 3 al 7 para la toma de las muestras de sangre con las líneas invertidas.

13. Volver a invertir las líneas, colocándolas en su posición normal de partida.

14. Aumentar el flujo de bomba a las cifras habituales que permita cada catéter.

15. Una vez analizadas las muestras en el laboratorio, con los datos obtenidos, calculamos el porcentaje 
(\%) de recirculación $(R)$, con las líneas en ambas posiciones (normal e invertida) según la fórmula:

\section{$R=B U N A 2-B U N A 1 / B U N A 2-B U N V \times 100^{1}$}

Las muestras sanguíneas se extrajeron una vez por semana, hasta un total de 4 en cada paciente.

Cualquier tipo de manipulación sobre los catéteres fue realizada siguiendo minuciosamente los protocolos de asepsia de la unidad.

\section{- Análisis estadístico}

Las variables cuantitativas fueron descritas como media \pm desviación estándar (DE) y su normalidad fue establecida con la prueba de Kolmogorov-Smirnov. Por su parte las variables cualitativas fueron descritas mediante frecuencias absolutas y relativas (porcentajes).

Para estudiar la diferencia entre medias entre dos grupos se utilizó la prueba de la U de Mann-Whitney. Para todas las pruebas utilizadas, se consideró un nivel de significación $p \leq 0,05$. El análisis estadístico se realizó con el programa SPSS v. 15.0

\section{- Aspectos éticos}

Declaración de Helsinki.

Se contó con la aprobación del Comité de Investigación del centro y de la Comisión de Ética e Investigación Clínica (CEIC).

Consentimiento informado.

\section{Resultados}

De los pacientes seleccionados, 5 eran mujeres $(41,6 \%)$ y 7 varones $(58,4 \%)$, con una edad media de 68,3 \pm 16,9 años.

El tiempo medio de permanencia de los catéteres era de 388,4 \pm 191 días.

La duración de las sesiones de HD fue de 222,5 \pm 20 minutos por sesión.
El protocolo de sellado que aplicamos a cada paciente fue el mismo que se les venía realizando con anterioridad, y se mantuvo así todo el tiempo que duró el estudio.

Los dializadores cuya superficie de membrana osciló entre 1,6-1,8 $\mathrm{m}^{2}$, también fueron los mismos que se utilizaban con anterioridad y que se mantuvieron a lo largo de todo el estudio.

El lugar de inserción de los catéteres es: Vena Yugular Interna Derecha: 10 pacientes $\left(83^{\prime} 3 \%\right)$, Vena Yugular Interna Izquierda: 1 paciente $\left(8^{\prime} 3 \%\right)$, Vena Femoral Izquierda: 1 paciente ( $83 \%$ ). (Fig. 1 ).

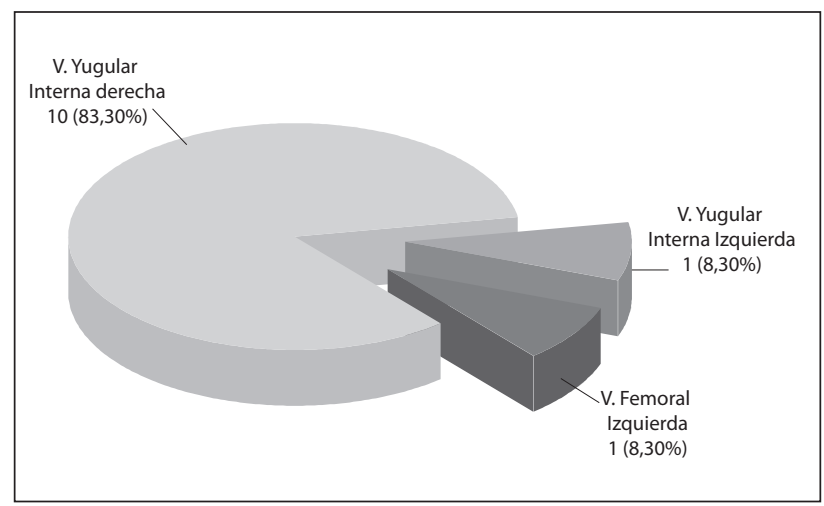

Figura 1.

Los resultados obtenidos tras el estudio de las sesiones de HD, fueron:

- Flujo medio de sangre fue de $339,4 \pm 12,3 \mathrm{ml} / \mathrm{min}$.

- Flujo del baño de diálisis se mantuvo siempre en $500 \mathrm{ml} / \mathrm{min}$

- La media de la presión arterial (PA) fue de -168,5 $\pm 24,9 \mathrm{~mm} \mathrm{Hg}$, y la de la presión venosa (PV) de $139,5 \pm 16,2 \mathrm{~mm} \mathrm{Hg}$.

- La media del Kt fue de 39,2 $\pm 4,7$ y la del Kt/v: 1,3 $\pm 0,2$

El número de infecciones fue de un solo caso, coincidiendo con una extrusión parcial del dacrón.

Del total de las 156 sesiones estudiadas, hubo que invertir las líneas en 8 ocasiones, en distintos pacientes, para mejorar los flujos, sin precisar en ningún momento la aplicación del protocolo de fibrinolíticos (urokinasa). 
La media de la recirculación con las líneas en posición normal fue de $4,5 \pm 2,9 \%$ y con las líneas en posición invertida de 4,6 $\pm 3,1 \%$. No existió diferencia estadísticamente significativa (NS) entre ambas medias. (Tabla 1).

\begin{tabular}{|l|l|}
\hline Flujo de sangre & $339,4 \pm 12,3 \mathrm{ml} / \mathrm{min}$ \\
\hline Flujo del baño & $500 \mathrm{ml} / \mathrm{min}$ \\
\hline Presión arterial & $-168,5 \pm 24,9 \mathrm{~mm} \mathrm{hg}$ \\
\hline Presión venosa & $139,5 \pm 16,2 \mathrm{~mm} \mathrm{hg}$ \\
\hline $\mathrm{Kt}$ & $39,2 \pm 4,7$ \\
\hline $\mathrm{Kt} / \mathrm{v}$ & $1,3 \pm 0,2$ \\
\hline $\mathrm{N}^{0}$ infecciones & 1 \\
\hline $\mathrm{N}^{0}$ inversión de líneas & 8 \\
\hline $\mathrm{N}^{\circ}$ protocolos urokinasa & 0 \\
\hline Recirculación normal & $4,5 \pm 2,9 \%$ \\
\hline Recirculación invertida & $4,6 \pm 3,1 \%$ \\
\hline
\end{tabular}

Tabla 1.

\section{Discusión}

La experiencia nos dice que cuando por necesidad se invierten las ramas de un catéter, el resultado es una diálisis de inferior calidad, debido a una mayor recirculación, hecho ya comprobado a través de múltiples estudios realizados con distintos tipos de catéteres, lo que de alguna manera ha llevado a los investigadores a diseñar catéteres que eviten o disminuyan en lo posible este problema, obteniendo importantes avances. Así, éstos fueron pasando de tener una sola luz, a través de la cual se realizaba la HD siguiendo el método de unipunción ${ }^{1}$, (la sangre salía y retornaba a través de esa única luz del catéter, con el consiguiente grado de recirculación que eso implicaba), a tener doble luz, con los que ya se realizaba la HD con el método de bipunción (la sangre sale por una rama y retorna por la otra), con lo cual el grado de recirculación disminuyó considerablemente aunque no todo lo que sería deseable. Otro punto importante, objeto de estudio por parte de los investigadores, era el diseño de las puntas.
Así fueron apareciendo en el mercado los catéteres con puntas de distinta longitud (escalonadas), los catéteres gemelos que se insertan en el mismo vaso sanguíneo (tipo Tesio), con puntas separadas (tipo Split), con puntas simétricas en disposición espiroidal (tipo palindrómicos) ${ }^{2,3}$, etc., por un afán de conseguir mejores prestaciones para mejorar la calidad de la HD y evitar en lo posible la recirculación de la sangre al aplicar esta técnica.

Cierto es que en la obtención de una HD de calidad influyen innumerables factores: tipo de dializador, horas de HD, acceso vascular, localización del catéter, de las puntas, disfunciones, infecciones etc. e incluso, nos atrevemos a decir que aún manteniendo las mismas condiciones y parámetros de la técnica en la misma persona, no siempre se consigue la misma calidad en todas las sesiones.

La escasez de trabajos publicados en catéteres de nueva generación, que aborden el tema de la recirculación, e incluso, la ausencia de trabajos realizados con pacientes portadores de este tipo de catéteres durante la sesión de HD, (los publicados están basados en estudios en animales ${ }^{6}$ ), unidos al hecho de que éstos son los que se implantan a nuestros pacientes, fue lo que nos animó a estudiar un parámetro sobre el que no podemos actuar y que, sin embargo, sí repercute en la calidad de la HD, como es la recirculación de la sangre en estos catéteres. Hemos estudiado dicha recirculación mediante el método ya explicado de la determinación sérica de la urea, ya que no disponemos de dispositivos para poder determinarla por otros medios (técnicas de dilución, ultrasonografía, etc.).

\section{Conclusiones}

Podemos concluir que los catéteres de las características previamente citadas presentan un grado de recirculación con las líneas en posición normal de $4^{\prime} 5 \pm 2^{\prime} 9 \%$ y de 4,6 3,1\% con líneas en posición invertida, (Fig. 2) cifras más que aceptables para poder conseguir HD de buena calidad, ya que está muy por debajo de la cifra que recomiendan las guías de la SEN de accesos vasculares ( $<$ de un $5 \%$ con técnicas de dilución y $<$ de un $10 \%$ con la determinación sérica de la urea). (Fig. 3). 


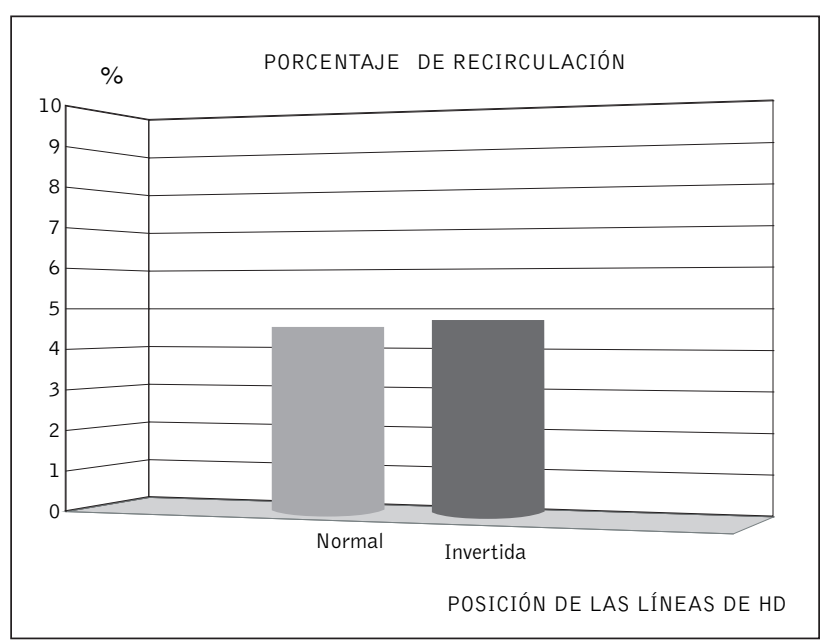

Figura 2.

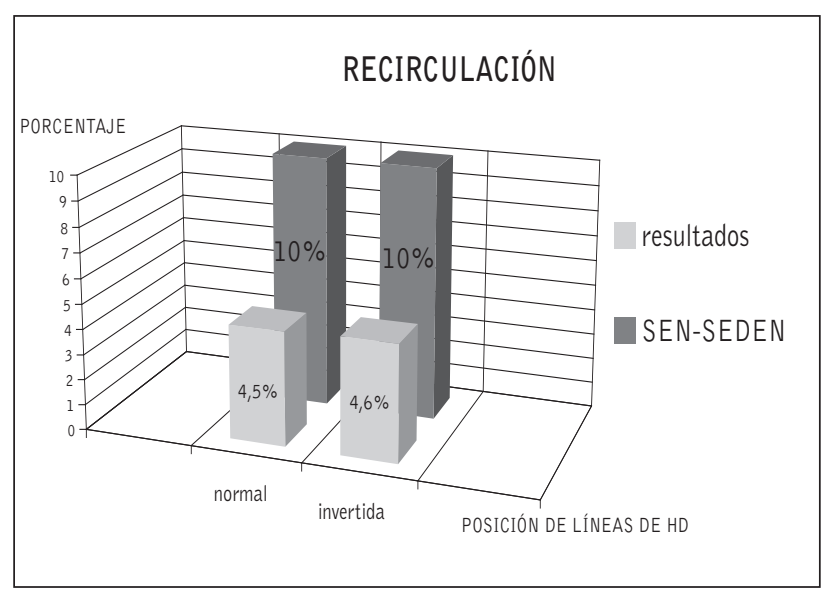

Figura 3.

Del mismo modo, podemos afirmar que los catéteres utilizados por los pacientes de nuestra unidad, incluidos en el estudio, presentan prácticamente la misma recirculación con las líneas del circuito en posición normal que con ellas en posición invertida, lo cual nos da una cierta garantía en cuanto a conseguir un HD eficaz y de calidad, incluso invirtiendo las líneas.

Somos conscientes de que la muestra empleada es muy pequeña, por lo que creemos que deben realizarse más estudios aleatorios para poder generalizar estas conclusiones.

\section{Agradecimientos}

A los pacientes por su consentimiento.

A todo el personal que nos ayudó en la realización de este trabajo.

Recibido: 23 Septiembre 2011

Revisado: 27 0ctubre 2011

Modificado: 16 Enero 2012

Aceptado: 27 Enero 2012

\section{Bibliografía}

1. Rodriguez JA, González E, Gutiérrez JM, Segarra A, Almirante B, Martínez MT et al. Guías SEN. Guías de acceso vascular en hemodiálisis. Nefrol. 2005; XXV(sup. 1).

2. Solozábal C. Utilización de catéteres y tipos. En: González MT, Martínez R. Manual de accesos vasculares para HD. Valencia: Marge Médica Books; 2010: 75-83.

3. Ash SR. Advances in tunneled central venous catheters for dialysis: design and performance. Seminars in Dialysis. 2008; 21(6): 504-515.

4. III Nacional Kidney Foundation. K/DOQI Clinical Practice Guidelines for Vascular Access, 2000. Guideline 12: Recirculation Methodology, Limits, Evaluation, on Follow-up.

5. Twardowski ZJ, Van Stone JC, Jones ME, Klusmeyer ME and Haynie JD. Blood recirculación in intravenous catheters for hemodialysis. J Am Soc Nephrol. 1993; 3(12): 1978-1981.

6. Tal MG. Comparison of recirculation percentage of the Palindrome catheter and standard hemodialysis catheters in a swine model. J Vasc Interv Radiol. 2005; 16 (9): 1237-1240. 